\title{
Monotonicity properties for the viable control of discrete-time systems
}

\author{
Michel De Lara ${ }^{\mathrm{a},{ }^{*}}$, Luc Doyen $^{\mathrm{b}}$, Thérèse Guilbaud $^{\mathrm{c}}$ and Marie-Joëlle Rochet ${ }^{\mathrm{c}}$
}

a CERMICS, Ecole des Ponts, ParisTech, 6-8 avenue Blaise Pascal, Champs sur Marne, 77455 Marne la Vallée Cedex 2, France

b CNRS, CERESP (UMR 5173 CNRS-MNHN-P6), Muséum National d'Histoire Naturelle, 55 rue Buffon, 75005 Paris, France

c Département EMH (Ecologie et Modèles pour l'Halieutique), IFREMER, B.P. 21105, 44311 Nantes

Cedex 03, France

*: Corresponding author : delara@cermics.enpc.fr

\begin{abstract}
:
This paper deals with the control of nonlinear systems in the presence of state and control constraints for discrete-time dynamics in finite-dimensional spaces. The viability kernel is known to play a basic role for the analysis of such problems and the design of viable control feedbacks. Unfortunately, this kernel may display very nonregular geometry and its computation is not an easy task in general. In the present paper, we show how monotonicity properties of both dynamics and constraints allow for relevant analytical upper and lower approximations of the viability kernel through weakly and strongly invariant sets. An example on fish harvesting management illustrates some of the assertions.
\end{abstract}

Keywords: Control; State constraints; Viability; Invariance; Monotonicity 


\title{
Monotonicity properties for the viable control of discrete time systems
}

\author{
Michel De LarA, Luc Doyen† Thérèse Guilbaud ${ }^{\ddagger}$ Marie-Joëlle RocheT ${ }^{\ddagger}$
}

April 24, 2007

\begin{abstract}
This paper deals with the control of nonlinear systems in the presence of state and control constraints for discrete time dynamics in finite dimensional spaces. The viability kernel is known to play a basic role for the analysis of such problems and the design of viable control feedbacks. Unfortunately, this kernel may display very non regular geometry and its computation is not an easy task in general. In the present paper, we show how monotonicity properties of both dynamics and constraints allow for relevant analytical upper and lower approximations of the viability kernel through weakly and strongly invariant sets. An example on fish harvesting management illustrates some of the assertions.
\end{abstract}

Key words: control, state constraints, viability, invariance, monotonicity.

\section{Introduction}

Let us consider a nonlinear control system described in discrete time by the difference equation

$$
\left\{\begin{array}{l}
x_{t+1}=f\left(x_{t}, u_{t}\right), \quad \forall t \in \mathbb{N} \\
x_{0} \text { given, }
\end{array}\right.
$$

where the state variable $x_{t}$ belongs to the finite dimensional state space $\mathbb{X}=\mathbb{R}^{n_{\mathbb{X}}}$, the control variable $u_{t}$ is an element of the control set $\mathbb{U}=\mathbb{R}^{n_{\mathbb{U}}}$ while the dynamics $f$ maps $\mathbb{X} \times \mathbb{U}$ into $\mathbb{X}$.

A controller or a decision maker describes "desirable configurations of the system" through a set $\mathbb{D} \subset \mathbb{X} \times \mathbb{U}$ termed the desirable set

$$
\left(x_{t}, u_{t}\right) \in \mathbb{D}, \quad \forall t \in \mathbb{N}
$$

where $\mathbb{D}$ includes both system states and controls constraints. Typical instances of such a desirable set are given by inequalities requirements: $\mathbb{D}=\left\{(x, u) \in \mathbb{X} \times \mathbb{U} \mid \forall i=1, \ldots, p, \quad g_{i}(x, u) \geq 0\right\}$.

\footnotetext{
${ }^{*}$ CERMICS, Ecole des Ponts, ParisTech, 6-8 avenue Blaise Pascal Champs sur Marne - 77455 Marne la Vallée Cedex 2 - France. Corresponding author: delara@cermics.enpc.fr, fax +33164153586

${ }^{\dagger}$ CNRS, CERESP (UMR 5173 CNRS-MNHN-P6), Muséum National d'Histoire Naturelle, 55 rue Buffon, 75005 Paris, France

${ }^{\ddagger}$ Département EMH (Ecologie et Modèles pour l'Halieutique), IFREMER, B.P. 21105, 44311 Nantes Cedex 03, France
} 
The state constraints set associated with $\mathbb{D}$ is obtained by projecting the desirable set $\mathbb{D}$ onto the state space $\mathbb{X}$ :

$$
\mathbb{V}^{0}:=\operatorname{Proj}_{\mathbb{X}}(\mathbb{D})=\{x \in \mathbb{X} \mid \exists u \in \mathbb{U},(x, u) \in \mathbb{D}\} .
$$

Such problems of dynamic control under constraints refers to viability [1] or invariance [11] framework. Basically, such an approach focuses on inter-temporal feasible paths. It has been applied for instance to models related to the sustainable management of resource and bio-economic modeling as in $[3,4,5,12,15,16,19]$. From the mathematical viewpoint, most of viability and weak invariance results are addressed in the continuous time case. However, some mathematical works deal with the discrete-time case. This includes the study of numerical schemes for the approximation of the viability problems of the continuous dynamics as in $[1,17]$. Important contributions for the discrete time case are also captured by the study of the positivity for linear systems as in [6], or by the hybrid control as in $[2,20]$. In the control theory literature, problems of constrained control lead to the study of positively invariant sets, particularly ellipsoidal and polyhedral ones for linear systems (see $[9,13,14]$ and the survey paper [10]); reachability of target sets or tubes for nonlinear discrete time dynamics is examined in [7].

Viability is defined as the ability to choose, at each time step $t \in \mathbb{N}$, a control $u_{t} \in \mathbb{U}$ such that the system configuration remains desirable. More precisely, the system is viable if the following feasible set is not empty:

$$
\mathbb{V}(f, \mathbb{D}):=\left\{\begin{array}{l|l}
x_{0} \in \mathbb{X} & \begin{array}{l}
\exists\left(u_{0}, u_{1}, \ldots\right) \text { and }\left(x_{0}, x_{1}, \ldots\right) \\
\text { satisfying }(1) \text { and }(2)
\end{array}
\end{array}\right\}
$$

The set $\mathbb{V}(f, \mathbb{D})$ is called the viability kernel associated with the dynamics $f$ and the desirable set $\mathbb{D}$. By definition, we have $\mathbb{V}(f, \mathbb{D}) \subset \mathbb{V}^{0}=\operatorname{Proj}_{\mathbb{X}}(\mathbb{D})$ but, in general, the inclusion is strict. For a decision maker or control designer, knowing the viability kernel has practical interest since it describes the states from which controls can be found that maintain the system in a desirable configuration forever. However, computing this kernel is not an easy task in general.

The present paper aims at giving explicit upper and lower approximations of this kernel using weakly (viable) or strongly invariant domains in the specific context of monotonicity properties of both constraints and dynamics ${ }^{1}$. To achieve this, let us recall what is meant by weakly or strongly invariant domains.

A subset $\mathbb{V}$ of the state space $\mathbb{X}$ is said to be strongly invariant for the dynamics $f$ in the desirable set $\mathbb{D}$ if

$$
\forall x \in \mathbb{V}, \quad \forall u \in \mathbb{U}, \quad(x, u) \in \mathbb{D} \Longrightarrow f(x, u) \in \mathbb{V} .
$$

That is, if one starts from $\mathbb{V}$, any control may transfer the state in $\mathbb{V}$ into a desirable configuration. This is generally a too demanding requirement.

Similarly, a subset $\mathbb{V}$ is said to be weakly invariant for the dynamics $f$ in the desirable set $\mathbb{D}$, or a viability domain of $f$ in $\mathbb{D}$, if

$$
\forall x \in \mathbb{V}, \quad \exists u \in \mathbb{U}, \quad(x, u) \in \mathbb{D} \text { and } f(x, u) \in \mathbb{V} .
$$

That is, if one starts from $\mathbb{V}$, a suitable control may transfer the state in $\mathbb{V}$ and the system into a desirable configuration. In particular, it is worth pointing out that any desirable equilibrium is a

\footnotetext{
${ }^{1}$ No topological assumptions are needed. Only for Proposition 8 , do we require a continuity property.
} 
viability domain of $f$ in $\mathbb{D}$. A desirable equilibrium is an equilibrium of the system that belongs to $\mathbb{D}$, that is a pair $(\bar{x}, \bar{u}) \in \mathbb{D}$ such that $\bar{x}=f(\bar{x}, \bar{u})$.

Moreover, according to viability theory [1], the viability kernel $\mathbb{V}(f, \mathbb{D})$ turns out to be the union of all viability domains, that is the largest set such that

$$
\mathbb{V}(f, \mathbb{D})=\bigcup\left\{\mathbb{V}, \mathbb{V} \subset \mathbb{V}^{0}, \mathbb{V} \text { viability domain for } f \text { in } \mathbb{D}\right\} \text {. }
$$

For the sake of completeness, we recall the proof in the Appendix (see Proposition 12). A major interest of such a property lies in the fact that any viability domain for the dynamics $f$ in the desirable set $\mathbb{D}$ provides a lower approximation of the viability kernel.

An upper approximation $\mathbb{V}_{k}$ of the viability kernel is given by the so called viability kernel until time $k$ associated with $f$ in $\mathbb{D}$ :

$$
\mathbb{V}_{k}:=\left\{\begin{array}{l|l}
x_{0} \in \mathbb{X} & \begin{array}{l}
\exists\left(u_{0}, u_{1}, \ldots, u_{k}\right) \text { and }\left(x_{0}, x_{1}, \ldots, x_{k}\right) \\
\text { satisfying }(1) \text { for } t=0, \ldots, k-1 \\
\text { and (2) for } t=0, \ldots, k
\end{array}
\end{array}\right\}
$$

We have

$$
\mathbb{V}(f, \mathbb{D}) \subset \mathbb{V}_{k+1} \subset \mathbb{V}_{k} \subset \mathbb{V}_{0}=\mathbb{V}^{0}, \quad \forall k \in \mathbb{N} .
$$

It may be seen by induction that the decreasing sequence of viability kernels until time $k$ satisfies the following dynamic programming equation

$$
\mathbb{V}_{0}=\mathbb{V}^{0} \text { and } \mathbb{V}_{k+1}=\left\{x \in \mathbb{V}_{k} \mid \exists u \in \mathbb{U}, f(x, u) \in \mathbb{V}_{k} \text { and }(x, u) \in \mathbb{D}\right\} .
$$

By (9), such an algorithm provides approximation from above of the viability kernel as follows:

$$
\mathbb{V}(f, \mathbb{D}) \subset \bigcap_{k \in \mathbb{N}} \mathbb{V}_{k}=\lim _{k \rightarrow+\infty} \downarrow \mathbb{V}_{k}
$$

In [1], conditions for the equality to hold true are exposed (are required the compacity for the constraints and upper semicontinuity with closed images for the set-valued map associated with the controlled dynamics).

Once the viability kernel, or any approximation, or a viability domain is known, we have to consider the management or control issue, that is the problem of selecting suitable controls at each time step. For any viability domain $\mathbb{V}$ and any state $x \in \mathbb{V}$, the following subset $\mathbb{U}_{\mathbb{V}}(x)$ of the decision set $\mathbb{U}$ is not empty:

$$
\mathbb{U}_{\mathbb{V}}(x):=\{u \in \mathbb{U} \mid(x, u) \in \mathbb{D} \text { and } f(x, u) \in \mathbb{V}\} .
$$

Therefore $\mathbb{U}_{\mathbb{V}(f, \mathbb{D})}(x)$ stands for the largest set of viable controls associated with $x \in \mathbb{X}$. Then, the decision design consists in the choice of a viable feedback control, namely any selection $\Psi: \mathbb{X} \rightarrow \mathbb{U}$ which associates with each state $x \in \mathbb{V}(f, \mathbb{D})$ a control $u=\Psi(x)$ satisfying $\Psi(x) \in \mathbb{U}_{\mathbb{V}(f, \mathbb{D})}(x)$.

The paper is organized as follows. Section 2 is devoted to the definitions of monotonicity for both the dynamics and constraints. Then, Section 3 exhibits lower and upper approximations of the viability kernel in this monotonicity context. An example is exposed in Section 4 to illustrate some of the main findings. 


\section{Monotonicity properties}

In this section we define what is meant by monotonicity of the desirable set $\mathbb{D}$ together with the dynamics $f$, both with respect to state $x$ and control $u$.

\subsection{Set monotonicity}

In what follows, the state space $\mathbb{X}$ and the control space $\mathbb{U}$ are $\mathbb{X} \subset \mathbb{R}^{n_{\mathbb{X}}}$ and $\mathbb{U} \subset \mathbb{R}^{n_{\mathbb{U}}}$ supplied with the componentwise order: $x^{\prime} \geq x$ if and only if each component of $x^{\prime}$ is greater than or equal to the corresponding component of $x$ :

$$
x^{\prime} \geq x \Longleftrightarrow x_{i}^{\prime} \geq x_{i}, i=1, \ldots, n .
$$

We also define the maximum $x \vee x^{\prime}$ of $\left(x, x^{\prime}\right)$ as follows:

$$
x \vee x^{\prime}:=\left(x_{1} \vee x_{1}^{\prime}, \ldots, x_{n} \vee x_{n}^{\prime}\right)=\left(\max \left(x_{1}, x_{1}^{\prime}\right), \ldots, \max \left(x_{n}, x_{n}^{\prime}\right)\right) .
$$

We now define the monotonicity of constraint sets.

Definition 1 [Set monotonicity] We say that a set $S \subset \mathbb{X}$ is increasing if it satisfies the following property:

$$
\forall x \in S, \quad \forall x^{\prime} \in \mathbb{X}, \quad x^{\prime} \geq x \Rightarrow x^{\prime} \in S .
$$

We say that a set $K \subset \mathbb{X} \times \mathbb{U}$ is increasing if it satisfies the following property:

$$
\forall(x, u) \in K, \quad \forall x^{\prime} \in \mathbb{X}, \quad x^{\prime} \geq x \Rightarrow\left(x^{\prime}, u\right) \in K .
$$

A geometric characterization of set monotonicity is given equivalently by $S+\mathbb{R}_{+}^{n_{\mathbb{X}}} \subset S$ in the first case, and by $K+\mathbb{R}_{+}^{n_{\mathbb{X}}} \times\left\{0_{\mathbb{R}^{n_{U}}}\right\} \subset K$ in the second case (where state and control do not play the same role).

\subsection{Dynamics monotonicity}

Similarly, we define monotonicity for the dynamics as follows.

Definition 2 [Mapping monotonicity] We say that the dynamics $f: \mathbb{X} \times \mathbb{U} \rightarrow \mathbb{X}$ is increasing with respect to the state if it satisfies

$$
\forall\left(x, x^{\prime}, u\right) \in \mathbb{X} \times \mathbb{X} \times \mathbb{U}, \quad x^{\prime} \geq x \Rightarrow f\left(x^{\prime}, u\right) \geq f(x, u),
$$

and is decreasing with respect to the control if

$$
\forall\left(x, u, u^{\prime}\right) \in \mathbb{X} \times \mathbb{U} \times \mathbb{U}, \quad u^{\prime} \geq u \Rightarrow f\left(x, u^{\prime}\right) \leq f(x, u) .
$$




\subsection{Maximal and saturated dynamics}

We define the maximal dynamics by $\bigvee_{u \in \mathbb{U},(x, u) \in \mathbb{D}} f(x, u)$, for $x \in \mathbb{V}^{0}$. Since the dynamics $f$ has several components (except when $\mathbb{X} \subset \mathbb{R}), \bigvee_{u \in \mathbb{U},(x, u) \in \mathbb{D}} f(x, u)$ is generally not achieved by a common $\bar{u}$; this is why we introduce the notion of function "saturated at $x$ ".

Definition 3 The maximal dynamics $\check{f}$ is defined by

$$
\forall x \in \mathbb{V}^{0}, \quad \check{f}(x):=\bigvee_{u \in \mathbb{U},(x, u) \in \mathbb{D}} f(x, u) .
$$

We say that the maximal dynamics $\check{f}$ is saturated at $x \in \mathbb{V}^{0}$ if there exists $u \in \mathbb{U}$ such that $(x, u) \in \mathbb{D}$ and $\check{f}(x)=f(x, u)$.

When $\mathbb{X} \subset \mathbb{R}$ and under appropriate topological assumptions (such as $\mathbb{U}$ is compact and the dynamics $f$ is continuous with respect to the control), the maximal dynamics is saturated.

Lemma 4 Assume that the desirable set $\mathbb{D}$ is increasing and that the dynamics $f$ is increasing with respect to the state. Then the maximal dynamics $\check{f}$ is increasing with respect to the state, in the sense that

$$
\forall\left(x, x^{\prime}\right) \in \mathbb{V}^{0} \times \mathbb{V}^{0}, \quad x^{\prime} \geq x \Rightarrow \check{f}\left(x^{\prime}\right) \geq \check{f}(x) .
$$

Proof. We have:

$$
\begin{aligned}
x^{\prime} \geq x \Rightarrow & f\left(x^{\prime}, u\right) \geq f(x, u), \quad \forall u \in \mathbb{U} \text { since } f \text { is increasing with the state } \\
\Rightarrow & \bigvee_{u \in \mathbb{U},\left(x^{\prime}, u\right) \in \mathbb{D}} f\left(x^{\prime}, u\right) \geq \bigvee_{u \in \mathbb{U},\left(x^{\prime}, u\right) \in \mathbb{D}} f(x, u) \\
\Rightarrow & \bigvee_{u \in \mathbb{U},\left(x^{\prime}, u\right) \in \mathbb{D}} f\left(x^{\prime}, u\right) \geq \bigvee_{u \in \mathbb{U},\left(x^{\prime}, u\right) \in \mathbb{D}} f(x, u) \geq \bigvee_{u \in \mathbb{U},(x, u) \in \mathbb{D}} f(x, u) \\
& \text { since the set } \mathbb{D} \text { is increasing and thus }(x, u) \in \mathbb{D} \Rightarrow\left(x^{\prime}, u\right) \in \mathbb{D} \\
& \text { i.e. }\{u \in \mathbb{U},(x, u) \in \mathbb{D}\} \subset\left\{u \in \mathbb{U},\left(x^{\prime}, u\right) \in \mathbb{D}\right\} \\
\Rightarrow & f f\left(x^{\prime}\right) \geq \check{f}(x) \text { by }(13) .
\end{aligned}
$$

\section{$3 \quad$ Viability results under monotonicity properties}

This section exhibits lower and upper approximations of the viability kernel in this monotonicity context. We show that some monotonicity properties of the dynamics $f$ and of the desirable set $\mathbb{D}$ are transmitted to the associated viability kernel. This allows to compute or approximate the viability kernel through viability domains under suitable assumptions.

A first obvious monotonicity property is that any viability domain associated with $f$ in $\mathbb{D}$ is a viability domain for any $\mathbb{D}^{\prime}$ such that $\mathbb{D} \subset \mathbb{D}^{\prime}$. 
Proposition 5 If the desirable set $\mathbb{D}$ is increasing and the dynamics $f$ is increasing with respect to the state, then the associated viability kernel $\mathbb{V}(f, \mathbb{D})$ is an increasing set, as well as all the sets $\mathbb{V}_{k}, k \in \mathbb{N}$ given by (10).

Proof. Let us prove that the set $\mathbb{V}(f, \mathbb{D})$ is increasing (see Definition 1 ). Consider $x \in \mathbb{V}(f, \mathbb{D})$ and $x^{\prime} \geq x$. By definition (4), there exists two sequences $\left(u_{t}\right)_{t \in \mathbb{N}}$ in $\mathbb{U}$ and $\left(x_{t}\right)_{t \in \mathbb{N}}$ in $\mathbb{X}$ such that

$$
x_{0}=x, \quad x_{t+1}=f\left(x_{t}, u_{t}\right) \text { and }\left(x_{t}, u_{t}\right) \in \mathbb{D}, \quad \forall t \in \mathbb{N} .
$$

Since $f$ is increasing with respect to the state (see Definition 2), we can show by induction that the trajectory $\left(x_{t}^{\prime}\right)_{t \in \mathbb{N}}$ defined by

$$
x_{0}^{\prime}=x^{\prime} \text { and } x_{t+1}^{\prime}=f\left(x_{t}^{\prime}, u_{t}\right), \quad \forall t \in \mathbb{N}
$$

satisfies $x_{t}^{\prime} \geq x_{t}, \forall t \in \mathbb{N}$. Since the set $\mathbb{D}$ is increasing, we deduce from $\left(x_{t}, u_{t}\right) \in \mathbb{D}$ and $x_{t}^{\prime} \geq x_{t}$ that $\left(x_{t}^{\prime}, u_{t}\right) \in \mathbb{D}, \forall t \in \mathbb{N}$. Thus $x^{\prime} \in \mathbb{V}(f, \mathbb{D})$ and, finally, the set $\mathbb{V}(f, \mathbb{D})$ is increasing.

For the rest, the proof uses the characterization (10) of the sets $\left(\mathbb{V}_{k}\right)_{k \in \mathbb{N}}$. The set $\mathbb{V}_{0}=\mathbb{V}^{0}=\{x \in \mathbb{X} \mid$ $\exists u \in \mathbb{U}, \quad(x, u) \in \mathbb{D}\}$ in $(3)$ is increasing since the set $\mathbb{D}$ is supposed to be increasing. Now, assume that the set $\mathbb{V}_{k}$ is increasing and consider $x \in \mathbb{V}_{k+1}$ and $x^{\prime} \geq x$. By the definition (10) of $\mathbb{V}_{k+1}$, there exists $u \in \mathbb{U}$ such that $(x, u) \in \mathbb{D}$ and $f(x, u) \in \mathbb{V}_{k}$. On the one hand, since the set $\mathbb{D}$ is increasing, we obtain that $\left(x^{\prime}, u\right) \in \mathbb{D}$. On the other hand, since the dynamics $f$ is increasing with respect to the state, we have $f\left(x^{\prime}, u\right) \geq f(x, u)$ and, since the set $\mathbb{V}_{k}$ is increasing, we obtain that $f\left(x^{\prime}, u\right) \in \mathbb{V}_{k}$. Thus, by (10) we obtain that $x^{\prime} \in \mathbb{V}_{k+1}$, meaning that the set $\mathbb{V}_{k+1}$ is increasing. By induction, we conclude that $\mathbb{V}_{k}$ is increasing for all $k \in \mathbb{N}$.

\subsection{A first lower approximation of the viability kernel}

The following result is a corollary of Proposition 5.

Proposition 6 If the desirable set $\mathbb{D}$ is increasing and the dynamics $f$ is increasing with respect to the state, and if there exists a desirable equilibrium $(\bar{x}, \bar{u})$, then

1. the upper orthant $\{x \in \mathbb{X} \mid x \geq \bar{x}\}$ is a viability domain for $f$ in $\mathbb{D}$;

2. consequently $\{x \in \mathbb{X} \mid x \geq \bar{x}\} \subset \mathbb{V}(f, \mathbb{D})$.

\section{Proof.}

1. Since $(\bar{x}, \bar{u})$ is a desirable equilibrium, the set $\{\bar{x}\}$ is a viability domain. The assumptions of Proposition 5 being satisfied, we obtain that the set $\{x \in \mathbb{X} \mid x \geq \bar{x}\}$ is a viability domain for $f$ in $\mathbb{D}$.

2. The viability kernel $\mathbb{V}(f, \mathbb{D})$ is the union of all viability domains by Proposition 12 . 


\subsection{A first upper approximation of the viability kernel}

We first exhibit strongly invariant domains, then provide an upper approximation of the viability kernel.

Proposition 7 Assume that the desirable set $\mathbb{D}$ is increasing and that the dynamics $f$ is increasing with respect to the state. Then the domain $\{x \in \mathbb{X} \mid x \leq \bar{x}\}$ is strongly invariant, whenever $\bar{x} \in \mathbb{V}^{0}$ satisfies $\check{f}(\bar{x}) \leq \bar{x}$ (in particular when $\bar{x}$ is a fixed point of $\check{f}$ ).

Proof. By Lemma 4 , the maximal dynamics $\check{f}$ is increasing with respect to the state. Let $\bar{x} \in \mathbb{V}^{0}$ satisfy $\check{f}(\bar{x}) \leq \bar{x}$. Let $x \in \mathbb{X}$ and $u \in \mathbb{U}$ be such that $x \leq \bar{x}$ and $(x, u) \in \mathbb{D}$. We have

$$
\begin{aligned}
f(x, u) & \leq \check{f}(x) \text { by }(13) \\
& \leq \check{f}(\bar{x}) \text { by Lemma } 4 \text { since } x \leq \bar{x} \\
& \leq \bar{x} \text { by assumption. }
\end{aligned}
$$

We have proved that

$$
x \leq \bar{x} \Rightarrow(\forall u \in \mathbb{U}, \quad(x, u) \in \mathbb{D} \Rightarrow f(x, u) \leq \bar{x}) .
$$

This means, by (5), that the domain $\{x \in \mathbb{X} \mid x \leq \bar{x}\}$ is strongly invariant for the dynamics $f$ in the desirable set $\mathbb{D}$.

Only for the following Proposition do we need to make a topological assumption.

Proposition 8 Assume that the desirable set $\mathbb{D}$ is increasing and that the dynamics $f$ is increasing with respect to the state. Assume also that the maximal dynamics $\check{f}$ is continuous, and that $\mathbb{V}^{0}$ is bounded from below. Define $\mathbb{M}$ as the set of those elements which are larger than at least one fixed point of $\check{f}$ in the closure $\overline{\mathbb{V}^{0}}$ of the state constraints set:

$$
\mathbb{M}:=\left\{x \in \mathbb{X} \mid \exists x^{\prime} \in \overline{\mathbb{V}^{0}}, \quad \check{f}\left(x^{\prime}\right)=x^{\prime}, \quad x \geq x^{\prime}\right\} .
$$

Then

$$
\mathbb{V}(f, \mathbb{D}) \subset \mathbb{V}^{0} \backslash\left\{x \in \mathbb{V}^{0} \mid \check{f}(x) \leq x \text { or } x \notin \mathbb{M}\right\}
$$

Proof. By Lemma 4, the maximal dynamics $\breve{f}$ is increasing with respect to the state.

Now assume that there exists $x_{0} \in \mathbb{V}(f, \mathbb{D})$ such that $\breve{f}\left(x_{0}\right) \leq x_{0}$. We shall prove that necessarily $x_{0} \in \mathbb{M}$. Let $\left(u_{0}, u_{1}, \ldots\right)$ and $\left(x_{0}, x_{1}, \ldots\right)$ be such that $x_{t+1}=f\left(x_{t}, u_{t}\right)$ and $\left(x_{t}, u_{t}\right) \in \mathbb{D}$. Define $\check{x}_{0}=x_{0}$ and $\check{x}_{t+1}=\check{f}\left(\check{x}_{t}\right)$, for $t=1,2 \ldots$. First, we prove by induction that $x_{t} \leq \check{x}_{t}$. The equality holds for $t=0$. Assuming $x_{t} \leq \check{x}_{t}$, since the maximal dynamics $\check{f}$ is increasing with respect to the state, we obtain that

$$
x_{t+1}=f\left(x_{t}, u_{t}\right) \leq \check{f}\left(x_{t}\right) \leq \check{f}\left(\check{x}_{t}\right)=\check{x}_{t+1} .
$$

Sedond, we deduce from $x_{t} \leq \check{x}_{t}$ that $\check{x}_{t} \in \mathbb{V}^{0}$ since $x_{t} \in \mathbb{V}^{0}$ and since the set $\mathbb{V}^{0}$ is increasing by Proposition 5 . Third, since $\check{f}$ is increasing with respect to the state and since $\check{f}\left(x_{0}\right) \leq x_{0}$, we easily see by induction that the sequence $\left(\check{x}_{t}\right)_{t \in \mathbb{N}}$ is decreasing.

The decreasing sequence $\left(\check{x}_{t}\right)_{t \in \mathbb{N}}$ being in $\mathbb{V}^{0}$, it is bounded below by assumption and therefore converges to $\underline{x} \in \overline{\mathbb{V}^{0}}$ (componentwise). By continuity of $\breve{f}$, we have $\breve{f}(\underline{x})=\underline{x}$. Thus, by $x_{0} \geq \underline{x}$, we deduce that $x_{0} \in \mathbb{M}$.

We can relax the assumption that the maximal dynamics $\check{f}$ is continuous, if we replace $\mathbb{M}$ by $\left\{x \in \mathbb{X} \mid \exists x^{\prime} \in \overline{\mathbb{V}^{0}}, \quad \check{f}\left(x^{\prime}\right) \leq x^{\prime} \leq x\right\}$. 


\subsection{A second lower approximation of the viability kernel}

If we adapt the dynamic programming algorithm (10), we obtain a lower approximation of the viability kernel as follows.

Proposition 9 If $\mathbb{V}$ is a viability domain of $f$ in $\mathbb{D}$, then

$$
\widetilde{\mathbb{V}}=\{x \in \mathbb{X} \mid \exists u \in \mathbb{U},(x, u) \in \mathbb{D} \text { and } f(x, u) \in \mathbb{V}\}
$$

is a viability domain which contains $\mathbb{V}$. As a consequence

1. the induction

$$
\widetilde{\mathbb{V}}_{0}=\mathbb{V} \text { and } \widetilde{\mathbb{V}}_{k+1}=\left\{x \in \mathbb{X} \mid \exists u \in \mathbb{U},(x, u) \in \mathbb{D} \text { and } f(x, u) \in \widetilde{\mathbb{V}}_{k}\right\}, \quad \forall k \in \mathbb{N}
$$

generates an increasing sequence of viability domains;

2. and its limit is included in the viability kernel:

$$
\bigcup_{k \in \mathbb{N}} \widetilde{\mathbb{V}}_{k}=\lim _{k \rightarrow+\infty} \uparrow \widetilde{\mathbb{V}}_{k} \subset \mathbb{V}(f, \mathbb{D}) .
$$

Proof.

By the definition of a viability domain, we have $\mathbb{V} \subset \widetilde{\mathbb{V}}$. For $x \in \widetilde{\mathbb{V}}$, by definition, there exists $u \in \mathbb{U}$ such that $(x, u) \in \mathbb{D}$ and $f(x, u) \in \mathbb{V} \subset \widetilde{\mathbb{V}}$; that is, $\widetilde{\mathbb{V}}$ is a viability domain of $f$ in $\mathbb{D}$.

1. Straightforward consequence.

2. Consequence of Proposition 12 in the Appendix.

\subsection{A second upper approximation of the viability kernel}

We adapt the dynamic programming algorithm (10) to the case of saturated dynamics. We stress that, under nice monotonicity properties, we obtain an algorithm converging exactly to the viability kernel $\mathbb{V}(f, \mathbb{D})$.

Proposition 10 Assume that the desirable set $\mathbb{D}$ is increasing and that the dynamics $f$ is increasing with respect to the state. Assume also that the maximal dynamics $\check{f}$ is saturated at all $x \in \mathbb{V}^{0}$. Then

1. the decreasing sequence (10) satisfies the backward induction

$$
\mathbb{V}_{0}=\mathbb{V}^{0} \text { and } \mathbb{V}_{k+1}=\mathbb{V}_{k} \bigcap \check{f}^{-1}\left(\mathbb{V}_{k}\right), \quad \forall k \in \mathbb{N}
$$

2. any $\mathbb{V}_{k}$ is an upper approximation of the viability kernel for $k \in \mathbb{N}: \mathbb{V}(f, \mathbb{D}) \subset \mathbb{V}_{k}$ 
3. the decreasing sequence $\left(\mathbb{V}_{k}\right)_{k \in \mathbb{N}}$ converges to $\mathbb{V}(f, \mathbb{D})$ :

$$
\mathbb{V}(f, \mathbb{D})=\bigcap_{k \in \mathbb{N}} \mathbb{V}_{k}=\lim _{k \rightarrow+\infty} \downarrow \mathbb{V}_{k}
$$

\section{Proof.}

1. By the assumptions on $\mathbb{D}$ and $f, \mathbb{V}_{k}$ is an increasing set by Proposition 5 . Thus, on the one hand, we have

$$
\begin{aligned}
x \in \mathbb{V}_{k+1} & \Longleftrightarrow \quad x \in \mathbb{V}_{k} \text { and } \exists u \in \mathbb{U}, f(x, u) \in \mathbb{V}_{k} \text { and }(x, u) \in \mathbb{D} \text { by }(10) \\
& \Rightarrow \quad x \in \mathbb{V}_{k} \text { and } \bigvee_{u \in \mathbb{U},(x, u) \in \mathbb{D}} f(x, u) \in \mathbb{V}_{k} \text { since the set } \mathbb{V}_{k} \text { is increasing } \\
& \Rightarrow \quad x \in \mathbb{V}_{k} \text { and } \check{f}(x) \in \mathbb{V}_{k} \text { by }(13) \\
& \Rightarrow \quad x \in \mathbb{V}_{k} \bigcap \check{f}^{-1}\left(\mathbb{V}_{k}\right) .
\end{aligned}
$$

On the other hand, let $x \in \mathbb{V}_{k} \cap \check{f}^{-1}\left(\mathbb{V}_{k}\right)$. Since $\mathbb{V}_{k} \subset \mathbb{V}^{0}$ and since, by assumption, the maximal dynamics $\check{f}$ is saturated at all $x \in \mathbb{V}^{0}$, let us denote by $\bar{u}$ an element of $\mathbb{U}$ such that $(x, \bar{u}) \in \mathbb{D}$ and $\check{f}(x)=f(x, \bar{u})$ (see Definition 3). We have:

$$
\begin{aligned}
x \in \mathbb{V}_{k} \bigcap \check{f}^{-1}\left(\mathbb{V}_{k}\right) & \Rightarrow x \in \mathbb{V}_{k} \text { and } f(x, \bar{u}) \in \mathbb{V}_{k} \text { and }(x, \bar{u}) \in \mathbb{D} \\
& \Rightarrow x \in \mathbb{V}_{k} \text { and } \exists u \in \mathbb{U}, f(x, u) \in \mathbb{V}_{k} \text { and }(x, u) \in \mathbb{D} \\
& \Rightarrow x \in \mathbb{V}_{k+1} \text { by }(10) .
\end{aligned}
$$

2. This follows from the previous item and from (9).

3. Let us denote $\mathbb{V}_{\infty}:=\lim _{k \rightarrow+\infty} \downarrow \mathbb{V}_{k}$ and prove that $\mathbb{V}(f, \mathbb{D})=\mathbb{V}_{\infty}$. Thanks to (11), we already know that $\mathbb{V}(f, \mathbb{D}) \subset \mathbb{V}_{\infty}$. We obtain the reverse inclusion $\mathbb{V}(f, \mathbb{D}) \supset \mathbb{V}_{\infty}$ by showing that $\mathbb{V}_{\infty}$ is a viability domain associated with $f$ in $\mathbb{D}$ (see Proposition 12). Consider a fixed $x \in \mathbb{V}_{\infty}$. Since the maximal dynamics $\check{f}$ is saturated at $x$, there exists $u \in \mathbb{U}$ such that $(x, u) \in \mathbb{D}$ and $\check{f}(x)=f(x, u)$. We claim that $f(x, u) \in \mathbb{V}_{k}$ for all $k \in \mathbb{N}$. For this, notice that, by (10) for all $k \in \mathbb{N}$, there exists $u_{k} \in \mathbb{D}$ such that $\left(x, u_{k}\right) \in \mathbb{D}$ and $f\left(x, u_{k}\right) \in \mathbb{V}_{k}$. On the one hand, by definition of the maximal dynamics $\check{f}$, we have $f\left(x, u_{k}\right) \leq \check{f}(x)=f(x, u)$. On the other hand, recall that $\mathbb{V}_{k}$ is an increasing set. Thus, $f(x, u) \in \mathbb{V}_{k}$. Since $\mathbb{V}_{\infty}=\bigcap_{k \in \mathbb{N}} \mathbb{V}_{k}$, we obtain that $f(x, u) \in \mathbb{V}_{\infty}$. We have proved that, for any $x \in \mathbb{V}_{\infty}$, there exists $u \in \mathbb{U}$ such that $(x, u) \in \mathbb{D}$ and $f(x, u) \in \mathbb{V}_{\infty}$. Hence $\mathbb{V}_{\infty}$ is a viability domain.

\subsection{A third lower approximation of the viability kernel}

A lower approximation of the viability kernel may be obtained by a lower approximation of the dynamics as follows.

Proposition 11 Assume that the desirable set $\mathbb{D}$ is increasing and that the dynamics $f$ is bounded below by an increasing $f^{b}: \mathbb{X} \times \mathbb{U} \rightarrow \mathbb{X}$ :

$$
\forall(x, u) \in \mathbb{X} \times \mathbb{U}, \quad f^{b}(x, u) \leq f(x, u) \text { and } f^{b} \text { is increasing with respect to the state. }
$$

Then, $\mathbb{V}\left(f^{b}, \mathbb{D}\right)$ is a viability domain associated with $f$ in $\mathbb{D}$, and thus

$$
\mathbb{V}\left(f^{b}, \mathbb{D}\right) \subset \mathbb{V}(f, \mathbb{D}) \text {. }
$$


Proof. The assumptions that the dynamics $f^{b}$ and the set $\mathbb{D}$ are increasing ensure that the set $\mathbb{V}\left(f^{b}, \mathbb{D}\right)$ is increasing, according to Proposition 5. Consider $x \in \mathbb{V}\left(f^{b}, \mathbb{D}\right)$. By Proposition 12, the viability kernel $\mathbb{V}\left(f^{b}, \mathbb{D}\right)$ is a viability domain for the dynamics $f^{b}$ : thus, there exists $u \in \mathbb{U}$ such that $(x, u) \in \mathbb{D}$ and $f^{b}(x, u) \in$ $\mathbb{V}\left(f^{b}, \mathbb{D}\right)$. By assumption $f(x, u) \geq f^{b}(x, u)$. Since $\mathbb{V}\left(f^{b}, \mathbb{D}\right)$ is increasing, we deduce that $f(x, u) \in \mathbb{V}\left(f^{b}, \mathbb{D}\right)$ : this means that $\mathbb{V}\left(f^{b}, \mathbb{D}\right)$ is a viability domain associated with the dynamics $f$ in $\mathbb{D}$.

Consequently, if $f$ is not well known, it can be replaced by an increasing sub-approximation which gives a viability domain associated with $f$ in $\mathbb{D}$. This may provide a deterministic and precautionary way of taking some uncertainty into account.

\section{An example}

In this section we apply various results to a model inspired by the management of an age structured abundance population model with a possibly non linear stock-recruitment relationship. This model is derived from fish stock management [18].

Let $A \in \mathbb{N}^{*}$ denote a maximum age, and $a \in\{1, \ldots, A\}$ an age class index. The state variable is $x=\left(x^{a}\right)_{a=1, \ldots, A} \in \mathbb{R}_{+}^{A}$, the abundances at age, and the control variable $u \in \mathbb{R}_{+}$is related to the harvest, so that $\mathbb{X}=\mathbb{R}_{+}^{A}$ and $\mathbb{U}=\mathbb{R}_{+}$with the notations of Section 1 . With $t$ the time index (years), the following dynamical relations relate the hereabove variables and define a dynamics $f$ (see $[18$, p. 256])

$$
\left\{\begin{array}{l}
x_{t+1}^{1}=\varphi\left(S S B\left(x_{t}\right)\right) \\
x_{t+1}^{a+1}=e^{-\left(M+u_{t} F^{a}\right)} x_{t}^{a}, \quad a \in\{1, \ldots, A-1\}
\end{array}\right.
$$

where $M \geq 0$ is the natural mortality, the non zero nonnegative vector $\left(F^{a}\right)_{a=1, \ldots, A-1}$ is the $e x$ ploitation pattern ${ }^{2}$ and $S S B$ is the spawning stock biomass, defined by ${ }^{3}$

$$
S S B(x):=\sum_{a=1}^{A} p^{a} w^{a} x^{a}
$$

and $\varphi$ describes a stock-recruitment relationship. We take the Beverton-Holt relationship [8]

$$
\varphi(x)=\frac{x}{\alpha+\beta x}
$$

which is increasing with respect to its argument. The exploitation is described by catch-at-age $C^{a}$ and yield $Y$, respectively defined for a given vector of abundance $x$ and a given control $u$ by the so called Baranov catch equations (see [18, p. 255-256]):

$$
C^{a}\left(x^{a}, u\right):=\frac{u F^{a}}{u F^{a}+M}\left(1-e^{-\left(M+u F^{a}\right)}\right) x^{a} \text { and } Y(x, u):=\sum_{a=1}^{A} w^{a} C^{a}\left(x^{a}, u\right)
$$

\footnotetext{
${ }^{2}$ This is why the control $u$ is called the exploitation pattern multiplier.

${ }^{3}$ Here, $\left(p_{a}\right)_{a=1, \ldots, A}$ are the proportions of mature individuals at age and $\left(w_{a}\right)_{a=1, \ldots, A}$ are the weights at age (all positive).
} 
State constraints set. The desirable set $\mathbb{D}$ we consider is defined by a minimum threshold $y_{\min }$ on the yield:

$$
\mathbb{D}:=\left\{(x, u) \in \mathbb{X} \times \mathbb{U} \mid Y(x, u) \geq y_{\min }\right\} .
$$

Since $u \mapsto C^{a}\left(x^{a}, u\right)$ is strictly increasing for at least one $a$ (recall that the exploitation pattern $\left(F^{a}\right)_{a=1, \ldots, A}$ is non zero nonnegative), the state constraints set $\mathbb{V}^{0}=\operatorname{Proj}_{\mathbb{X}}(\mathbb{D})$ is given by

$$
\mathbb{V}^{0}=\left\{x \in \mathbb{R}_{+}^{A} \mid \lim _{u \rightarrow+\infty} Y(x, u)>y_{\min }\right\}=\left\{x \in \mathbb{R}_{+}^{A} \mid \sum_{a=1, \ldots, A, F^{a}>0} w^{a} x^{a}>y_{\min }\right\} .
$$

Monotonicity properties. The desirable set is increasing, since $Y(x, u)$ is increasing with respect to $x$. The dynamics $f$ is increasing with respect to the state, since $\varphi$ is an increasing function, and is decreasing with respect to the control.

Computation of desirable equilibria. Introducing $D^{a}(u):=\mathrm{e}^{-\left((a-1) M+u\left(F^{1}+\ldots+F^{a-1}\right)\right)}(a=$ $2, \ldots, A)$, the proportion of equilibrium recruits which survive up to age $a\left(D_{1}(u)=1\right)$, and the equilibrium spawners per recruits

$$
\operatorname{spr}(u):=\sum_{a=1}^{A} p^{a} w^{a} D^{a}(u),
$$

we see that the function $x^{1} \mapsto \varphi\left(x^{1} \operatorname{spr}(u)\right)$, with $\varphi$ given by (22), has a nonnegative fixed point $R_{\varphi}(u)=\frac{1}{\beta}-\frac{\alpha}{\operatorname{spr}(u)}$, supposed to be positive, and which is decreasing with $u$. Then

$$
x^{*}(u):=\left(R_{\varphi}(u), D^{1}(u) R_{\varphi}(u), \ldots, D^{A}(u) R_{\varphi}(u)\right)
$$

is such that $f\left(x^{*}(u), u\right)=x^{*}(u)$, and is decreasing with $u$. There remains to find conditions under which such $\left(x^{*}(u), u\right)$ is not only an equilibrium but a desirable equilibrium, that is $Y\left(x^{*}(u), u\right) \geq$ $y_{\min }$.

For the sake of simplicity, we assume that the function $Y^{*}: u \mapsto Y\left(x^{*}(u), u\right)$ (yield at equilibrium) is continuous and goes to zero at infinity (we have $Y^{*}(0)=Y\left(x^{*}(0), 0\right)=0$ by (23)). Then, the function $Y^{*}$ admits a maximum value, commonly called the maximum sustainable yield, since it is the maximum equilibrium yield. We denote

$$
y_{\mathrm{msy}}:=\max _{u \geq 0} Y^{*}(u)=Y^{*}\left(u_{\mathrm{msy}}\right)
$$

achieved for a fishing effort multiplier $u_{\mathrm{msy}}$. By definition, $y_{\mathrm{msy}}$ is the maximum value for $y_{\min }$ such that there exists a desirable equilibrium.

Approximation of the viability kernel. Let us consider $\left.y_{\min } \in\right] 0, y_{m s y}[$. By the intermediate value theorem, there exist $u_{\text {min }}^{+}>u_{\text {min }}^{-}$such that $Y^{*}\left(u_{\text {min }}^{+}\right)=Y^{*}\left(u_{\min }^{-}\right)=y_{\text {min }}$. By the expression of $x^{*}(u)$, decreasing with $u$, we obtain that $x^{*}\left(u_{\min }^{+}\right) \leq x^{*}\left(u_{\min }^{-}\right)$. We define

$$
\widetilde{\mathbb{V}}^{-}=\left\{x \in \mathbb{R}_{+}^{A} \mid x \geq x^{*}\left(u_{\text {min }}^{-}\right)\right\} \subset \widetilde{\mathbb{V}}^{+}=\left\{x \in \mathbb{R}_{+}^{A} \mid x \geq x^{*}\left(u_{\text {min }}^{+}\right)\right\} .
$$

According to Proposition 6 , both sets $\widetilde{\mathbb{V}}^{+}$and $\widetilde{\mathbb{V}}^{-}$are viability domains associated with $f$ in $\mathbb{D}$, defined in $(24)$ with the threshold $y_{\min }$. We obtain the lower approximation of the viability kernel

$$
\widetilde{\mathbb{V}}^{+} \subset \mathbb{V}(f, \mathbb{D}) \text {. }
$$


The resulting patterns are shown in Figure 1 in the two-class case, that is with $A=2$. Using Proposition 9 , the viability domain obtained one step backward from $\widetilde{\mathbb{V}}^{+}$is sketched in Figure 2 .

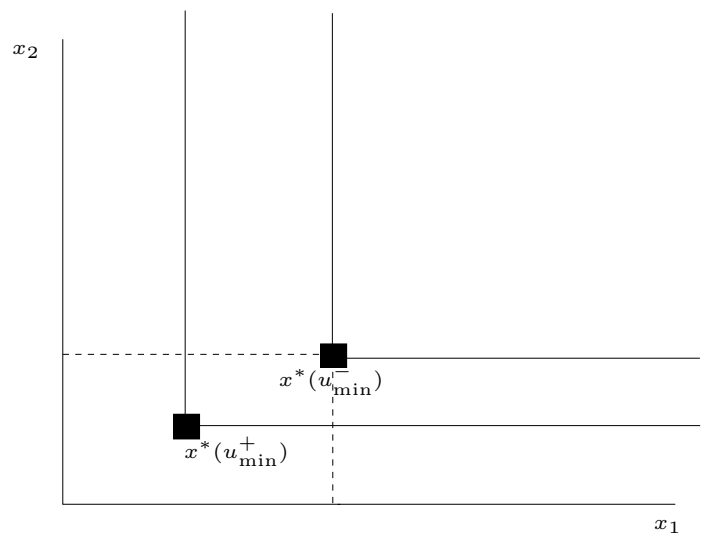

Figure 1: Example of two viability domains defined with two desirable equilibria.

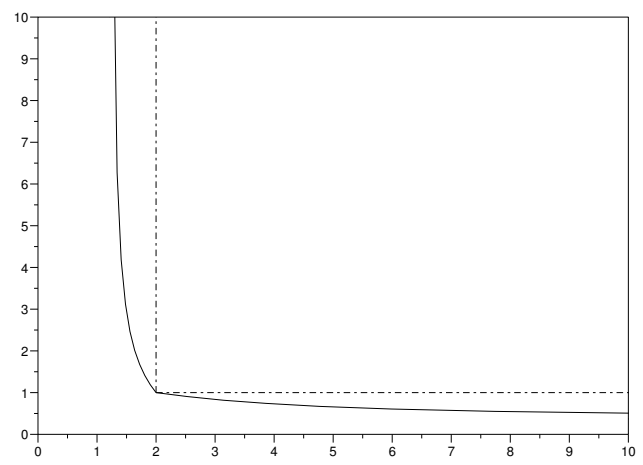

Figure 2: Enlargement of a viable orthant in the plan $\left(x^{1}, x^{2}\right)$.

Acknowledgement. We thank Lance Bode (James Cook University, Australia) for reading and commenting our paper.

\section{A Appendix}

Proposition 12 The viability kernel $\mathbb{V}(f, \mathbb{D})$ is the union of all viability domains, that is the largest set $\mathbb{V}$ such that $\mathbb{V} \subset\{x \in \mathbb{X} \mid \exists u \in \mathbb{U},(x, u) \in \mathbb{D}$ and $f(x, u) \in \mathbb{V}\}$. In other words, the viability kernel is the largest viability domain.

\section{Proof.}

First, we prove that any viability domain $\mathbb{V}$ associated with $f$ in $\mathbb{D}$ is a subset of $\mathbb{V}(f, \mathbb{D})$. For $x \in \mathbb{V}$, let us put $x_{0}=x$ and let $u_{0} \in \mathbb{U}$ be such that $x_{1}=f\left(x_{0}, u_{0}\right) \in \mathbb{V}$ and $\left(x_{0}, u_{0}\right) \in \mathbb{D}$. Starting from $x_{1}$, we 
proceed in the same way to obtain $u_{1}$. Going on, we find a sequence $\left(u_{0}, u_{1}, \ldots\right)$ such that $x_{t+1}=f\left(x_{t}, u_{t}\right)$ and $\left(x_{t}, u_{t}\right) \in \mathbb{D}$ for all $t \in \mathbb{N}$. Thus, $x \in \mathbb{V}(f, \mathbb{D})$.

Second, we prove that $\mathbb{V}(f, \mathbb{D})$ is a viability domain. By definition, for all $x \in \mathbb{V}(f, \mathbb{D})$, there exists decisions $u_{0}, u_{1} \ldots$ and states $x_{0}, x_{1} \ldots$ starting from $x$ at time 0 satisfying for all times $t \in \mathbb{N},\left(x_{t}, u_{t}\right) \in \mathbb{D}$ and $x_{t+1}=f\left(x_{t}, u_{t}\right)$. Let us set $y=f\left(x, u_{0}\right)$. With the states $y_{t}=x_{t+1}$ and the decisions $v_{t}=u_{t+1}$, we obtain that $y \in \mathbb{V}(f, \mathbb{D})$. Then, there exists $u\left(=u_{0}\right) \in \mathbb{U}$ such that $(x, u) \in \mathbb{D}$ and $f(x, u) \in \mathbb{V}(f, \mathbb{D})$. Thus, $\mathbb{V}(f, \mathbb{D})$ is a viability domain.

We conclude that $\mathbb{V}(f, \mathbb{D})$ is the largest viability domain associated with $f$ in $\mathbb{D}$ and that all the viability domains are included in $\mathbb{V}(f, \mathbb{D})$.

\section{References}

[1] J.-P. Aubin. Viability Theory. Birkhäuser, Boston, 1991.

[2] J.-P. Aubin, J. Lygeros, M. Quincampoix, S. Sastry, and N. Seube. Viability and invariance kernels of impulse differential inclusions. Decision and Control, 2:1639-1644, 2001.

[3] C. Béné and L. Doyen. Storage and viability of a fishery with resource and market dephased seasonnalities. J. of Environmental Resource Economics, 15:1-26, 2000.

[4] C. Béné and L. Doyen. Sustainability of fisheries through marine reserves: a robust modeling analysis. J. of Environmental Management, 69:1-13, 2003.

[5] C. Béné, L. Doyen, and D. Gabay. Viability analysis for a bio-economic model. Ecological Economics, 36:385-396, 2001.

[6] A. Berman and R.J. Plemmons. Nonnegative Matrices in the Mathematical Sciences, volume 9. Classics in Applied Mathematics, SIAM, Philadelphia, 1994.

[7] D. Bertsekas and I. Rhodes. On the minimax reachability of target sets and target tubes. Automatica, 7:233-247, 1971. citeseer.ist.psu.edu/bertsekas71minimax.html.

[8] R. J. H. Beverton and S. J. Holt. On the dynamics of exploited fish populations, volume 19 of Series II. Fishery Investigations. Her Majesty's Stationery Office, 1957. 533 pp.

[9] G. Bitsoris. On the positive invariance of polyhedral sets for discrete-time systems. Syst. Control Lett., 11(3):243-248, 1988.

[10] F. Blanchini. Set invariance in control (survey paper). Automatica, 35(11):1747-1767, 1999.

[11] F.H. Clarke, Y. S. Ledayev, R.J. Stern, and P.R. Wolenski. Qualitative properties of trajectories of control systems: a survey. J. of Dynamical Control Syst., 1:1-48, 1995.

[12] K. Eisenack, J. Sheffran, and J. Kropp. The viability analysis of management frameworks for fisheries. Environmental modelling and assessment, 11(1):69 - 79, February 2006.

[13] E.G. Gilbert and K.T. Tan. Linear systems with state and control constraints: the theory andapplication of maximal output admissible sets. IEEE Transactions on Automatic Control, 36(9):1008-1020, 1991. 
[14] P.-O. Gutman and M. Cwikel. Admissible sets and feedback control for discrete-time linear dynamical systems with bounded controls and states. IEEE Transactions on Automatic Control, 31(4):373- 376, 1986.

[15] V. Martinet and L. Doyen. Sustainable management of an exhaustible resource: a viable control approach. Journal of Resource and Energy Economics, forthcoming.

[16] C. Mullon, P. Cury, and L. Shannon. Viability model of trophic interactions in marine ecosystems. Natural Resource Modelling, 17:27-58, 2004.

[17] M. Quincampoix and P. Saint-Pierre. An algorithm for viability kernels in hölderian case: approximation by discrete dynamical systems. Journal of Mathematical Systems Estimation and Control, 5:1-13, 1995.

[18] T.J. Quinn and R.B. Deriso. Quantitative Fish Dynamics. Oxford University Press, 1999.

[19] A. Rapaport, J. P. Terreaux, and L. Doyen. Sustainable management of renewable resource: a viability approach. Mathematics and Computer Modeling, 43(5-6):466-484, March 2006.

[20] R. Vidal, S. Schaffert, J. Lygeros, and S. Sastry. Controlled invariance of discrete time systems. In In Hybrid Systems: Computation and Control, Lecture Notes in Computer Science, Springer Verlag, volume 1790, pages 437-450, 2000. 